\title{
BOUNDING THE DEGREE OF SOLUTIONS TO PFAFF EQUATIONS
}

\author{
Marco Brunella and Luís Gustavo Mendes
}

\begin{abstract}
We study hypersurfaces of complex projective manifolds which are invariant by a foliation, or more generally which are solutions to a Pfaff equation. We bound their degree using classical results on logarithmic forms.
\end{abstract}

\section{Introduction}

Starting with Poincaré $[\mathbf{P o}]$ and Painlevé $[\mathbf{P a}]$, many mathematicians considered the following problem: given a foliation $\mathcal{F}$ on $\mathbf{C} P^{n}$ of degree $d$ and a hypersurface $V \subset \mathbf{C} P^{n}$ invariant by $\mathcal{F}$, is it possible to bound the degree of $V$ by a number $h(d)$ which depends only on $d$ (and not on $\mathcal{F}$ )? In such a generality, the answer is clearly negative: for example, the curve $\left\{x^{p}=y^{q}\right\}$ in $\mathbf{C} P^{2}$ has degree $\max \{p, q\}$ and it is invariant by the foliation given by $p y d x-q x d y=0$, whose degree is one (see also $[\mathbf{L N}]$ for other interesting examples). However several positive results have been obtained by those authors and, recently, by $[\mathbf{C L}],[\mathbf{C a}],[\mathbf{B a}],[\mathbf{B r}]$ and $[\mathbf{S o}]$. The philosophy behind these results is that the failure of an uniform bound $h(d)$ is due to the existence of "bad singularities" of $V$ or of $\mathcal{F}$. For instance, one finds the bound $h(d)=d+2$ in $\mathbf{C} P^{2}$, provided that either $V$ has only normal crossing singularities $[\mathbf{C L}]$, or $\mathcal{F}$ has only nondicritical singularities along $V[\mathbf{C a}]$. Our aim is to attract the attention of the reader to the relation between this problem and these results and some basic properties of logarithmic forms, discovered by Deligne and Bogomolov [De], [Bo].

We shall work in the context of Pfaff equations, more general than foliations. For our purposes, the simplest definition is the following:

Definition 1.1. Given a complex manifold $X$ and a holomorphic line bundle $N$ on $X$, a Pfaff equation of codimension $p, 1 \leq p \leq \operatorname{dim}_{\mathbf{C}}(X)-1$, is a nontrivial global section $\sigma$ of $\Omega_{X}^{p} \otimes N$, where $\Omega_{X}^{p}$ denotes the sheaf of holomorphic $p$-forms on $X$. 
Let us consider the corresponding generalization of the notion of leaf of a foliation. Given a hypersurface $V$, let $i_{V} \sigma$ denote the restriction of $\sigma$ to $V$, i.e. the section of $\left.\Omega_{V}^{p} \otimes N\right|_{V}$ obtained by projecting $\left.\sigma\right|_{V}$ via $\left.\left.\left(\Omega_{X}^{p} \otimes N\right)\right|_{V} \rightarrow \Omega_{V}^{p} \otimes N\right|_{V}$.

Definition 1.2. Given a Pfaff equation $\sigma \in H^{0}\left(X, \Omega_{X}^{p} \otimes N\right)$ and a hypersurface $V \subset X$, we shall say that $V$ is a solution to $\sigma$ if $i_{V} \sigma \equiv 0$.

Some remarks on these definitions may be useful. Usually one requires that the zero set $(\sigma)_{0}$ of a Pfaff equation $\sigma \in H^{0}\left(X, \Omega_{X}^{p} \otimes N\right)$ has codimension $\geq 2$, but this is not really important. Anyway, if $\sigma \in$ $H^{0}\left(X, \Omega_{X}^{p} \otimes N\right)$ vanishes on a hypersurface $Z \subset X$, then we can replace $\sigma$ by $\sigma^{\prime}:=\frac{\sigma}{h} \in H^{0}\left(X, \Omega_{X}^{p} \otimes N^{\prime}\right)$, where $N^{\prime}=N \otimes \mathcal{O}_{X}(-Z)$ and $h \in$ $H^{0}\left(X, \mathcal{O}_{X}(Z)\right)$ vanishes on $Z$, and then $\left.\sigma^{\prime}\right|_{Z} \not \equiv 0$. This division of $\sigma$ by $h$ has no significant consequences, because in the study of Pfaff equations one is more interested in the saturated subsheaf of $\Omega_{X}^{p}$ generated by $\sigma$ than in $\sigma$ itself, and this subsheaf is unchanged by the division.

A foliation $\mathcal{F}$ of codimension $p$ gives rise to a Pfaff equation of codimension $p$, because locally such a foliation can be seen as the kernel of a holomorphic $p$-form; in this case, $N$ corresponds to the determinant line bundle of the rank $p$ normal sheaf of $\mathcal{F}$. But the converse is not generally true, except when $p=\operatorname{dim}_{\mathbf{C}}(X)-1$, because no integrability assumption is done. When the Pfaff equation arises from a foliation $\mathcal{F}$, Definition 1.2 reduces to say that the hypersurface $V$ is saturated by the leaves of $\mathcal{F}$.

In order to state our result in a simple form, let us suppose now that $X$ is a projective manifold with $\operatorname{Picard} \operatorname{group} \operatorname{Pic}(X)=\mathbf{Z}$, although a more general fact, without restrictions on $\operatorname{Pic}(X)$, will be found in the course of the proof. Let $H$ be the positive generator of $\operatorname{Pic}(X)$. We naturally define the degree of a holomorphic line bundle $L$ as the integer $d(L)$ such that $L=d(L) H$ in $\operatorname{Pic}(X)$. For a hypersurface $V$, the degree $d(V)$ is defined as $d\left(\mathcal{O}_{X}(V)\right)$. We recall that projective manifolds with $\operatorname{Pic}(X)=\mathbf{Z}$ are quite abundant: for instance, the classical NoetherLefschetz theorem states that a generic hypersurface $X$ in $\mathbf{C} P^{n}$ of high degree has $\operatorname{Pic}(X)=\mathbf{Z}$. We refer to Section 2 for the basic properties of logarithmic forms.

Theorem. Let $X$ be a complex projective manifold with $\operatorname{Pic}(X)=\mathbf{Z}$ and let $\sigma \in H^{0}\left(X, \Omega_{X}^{p} \otimes N\right)$ be a Pfaff equation. Let $V \subset X$ be a normal crossing hypersurface, which is solution to $\sigma$. Then

$$
d(V) \leq d(N)
$$

and the inequality is strict if $V$ is smooth. Moreover, if $d(V)=d(N)$ then $\sigma$ is given by a global closed logarithmic p-form on $X$ with poles along $V$. 
When $\operatorname{dim}_{\mathbf{C}}(X)=2$, we recover results of $[\mathbf{C L}]$ and $[\mathbf{B a}]$. Let us consider the case when a Pfaff equation on $\mathbf{C} P^{n}$ arises from a foliation with $\operatorname{codim}_{\mathbf{C}} \operatorname{Sing}(\mathcal{F}) \geq 2$ (and $\operatorname{codim}_{\mathbf{C}}(\sigma)_{0} \geq 2$ ). One usually defines the degree $d(\mathcal{F})$ as the degree of the tangency set between the $(n-p)$-dimensional leaves of $\mathcal{F}$ and a generic linear subspace $\Pi \simeq \mathbf{C} P^{p}$ (remark that this tangency set is a hypersurface of $\Pi$ ). In Lemma 3.2 we show that $d(N)=d(\mathcal{F})+p+1$ and we recover the result of $[\mathbf{C L}]$ in $\mathbf{C} P^{2}$, since our bound becomes

$$
d(V) \leq d(\mathcal{F})+p+1
$$

At the end of the paper we shall discuss to which extent the normal crossing hypothesis on $V$ may be weakened.

Acknowledgements. We thank the hospitality of the C.R.M., Barcelona, where this work was completed. The second author is supported by a grant of "Conseil Régional de Bourgogne", France, and he thanks the attention of P. Sad and M. G. Soares.

\section{Logarithmic forms}

In this section we recall basic facts on logarithmic forms (see, for instance, $[\mathbf{S a}])$.

Let $X$ be a complex manifold and $V$ a hypersurface with at most normal crossing singularities. A logarithmic $p$-form on $X$ with poles along $V$ is a meromorphic $p$-form $\omega$ with polar set $(\omega)_{\infty} \subset V$ such that $\omega$ and $d \omega$ have at most simple poles along $V$. Equivalently, if $f=0$ is a local reduced equation of $V$, then $f \omega$ and $f d \omega$ are holomorphic. Obviously, this condition is equivalent to $f \omega$ and $d f \wedge \omega$ being holomorphic.

This definition can be localized on open sets of $X$; therefore we obtain a (coherent, analytic, locally free) sheaf on $X$, denoted $\Omega_{X}^{p}(\log V)$. Remark that everything makes sense even if $p=0$ or $p=n=\operatorname{dim}_{\mathbf{C}}(X)$, and $\Omega_{X}^{0}(\log V)=\mathcal{O}_{X}, \Omega_{X}^{n}(\log V) \simeq \Omega_{X}^{n} \otimes \mathcal{O}_{X}(V)$.

If $\left(z_{1}, \ldots, z_{n}\right)$ is a local coordinate system around $x=(0, \ldots, 0) \in V$ such that $V$ is locally expressed by $V=\left\{z_{1} \cdot \ldots \cdot z_{k}=0\right\}$, then $\bigoplus_{p=0}^{n} \Omega_{X}^{p}(\log V)$ is locally generated by holomorphic forms and $\left\{\frac{d z_{1}}{z_{1}}, \ldots, \frac{d z_{k}}{z_{k}}\right\}$. More precisely, every section $\omega$ of $\Omega_{X}^{p}(\log V)$ can be locally written as

$$
\omega=\omega_{0}+\sum_{j=1}^{k} \omega_{j} \wedge \frac{d z_{j}}{z_{j}}
$$

where $\omega_{0}$ is a holomorphic $p$-form and each $\omega_{j}$ is a local section of $\Omega_{X}^{p-1}(\log V)$. Remark that the exterior product of two logarithmic forms 
is still a logarithmic form. We also note that the existence of a decomposition like (1) is strongly dependent on the hypothesis that $V$ has only normal crossing singularities.

In particular, for every $j=1, \ldots, k$, we can locally decompose $\omega$ as

$$
\omega=\gamma_{j}+\eta_{j} \wedge \frac{d z_{j}}{z_{j}}
$$

where $\gamma_{j}$ is a local section of $\Omega_{X}^{p}(\log V), \eta_{j}$ is a local section of $\Omega_{X}^{p-1}(\log V)$ and moreover both $\gamma_{j}$ and $\eta_{j}$ do not contain $V_{j}:=\left\{z_{j}=0\right\}$ in their polar set. The decomposition (1), as well as (2), is not unique; however, a simple computation shows that the restriction of $\eta_{j}$ to $V_{j}$ is intrinsically defined by $\omega$, i.e. does not depend on the involved choices. Setting

$$
\Gamma_{j}:=V_{j} \cap\left(\cup_{i=1, i \neq j}^{k} V_{i}\right),
$$

which is a normal crossing hypersurface of $V_{j}$, we therefore have a well defined map

$$
\operatorname{Res}_{V_{j}}:\left.\omega \mapsto \eta_{j}\right|_{V_{j}},
$$

and $\left.\eta_{j}\right|_{V_{j}} \in \Omega_{V_{j}}^{p-1}\left(\log \Gamma_{j}\right)$ is called local residue of $\omega$ along $V_{j}$. Summing on $j$ and patching together these local constructions, we finally obtain the residue map

$$
\text { Res: } \Omega_{X}^{p}(\log V) \rightarrow \Omega_{\hat{V}}^{p-1}(\log \Gamma),
$$

where $\hat{V}$ is the normalization of $V$ and $\Gamma \subset \hat{V}$ is the normal crossing hypersurface induced by $V$ on $\hat{V}$. Note that the kernel of the residue map is exactly $\Omega_{X}^{p}$ and that there is an exact sequence

$$
0 \rightarrow \Omega_{X}^{p} \rightarrow \Omega_{X}^{p}(\log V) \rightarrow \Omega_{\hat{V}}^{p-1}(\log \Gamma) \rightarrow 0 .
$$

The next lemma was proved in $[\mathbf{D e}]$, as a by-product of a logarithmic Hodge decomposition, but an elementary proof was later found in [No]. For sake of completeness, we give a proof, which is even simpler than that of $[\mathrm{No}]$.

Lemma 2.1. $[\mathbf{D e}]$ Let $X$ be a complex projective manifold, $V \subset X$ a normal crossing hypersurface and $\omega$ a global logarithmic p-form with polar set contained in $V$. Then $\omega$ is closed.

Proof: In order to prove that a $p$-form is closed, it is sufficient to prove that the restriction of the $p$-form to a generic $(p+1)$-dimensional submanifold is closed. Hence we may assume that $n=\operatorname{dim}_{\mathbf{C}}(X)=p+1$, and the proof will be by induction on $p$. 
The case $p=0$ is trivial: $\Omega_{X}^{0}(\log V)=\mathcal{O}_{X}$ and a global holomorphic function is constant. Assume now that the lemma has been proved for $(p-1)$-forms.

If $\omega \in H^{0}\left(X, \Omega_{X}^{p}(\log V)\right)$, then, as in [No], we may consider the current $T_{\omega}$ of bidegree $(p, 0)$ defined by

$$
T_{\omega}(\phi)=\int_{X} \omega \wedge \phi,
$$

for every smooth $(1, p+1)$-form $\phi$. This is well defined, i.e. the integral is convergent, precisely because $\omega$ has logarithmic poles along $V$ : the 2 -form $\frac{1}{z} d z \wedge d \bar{z}$ is integrable on the disc. Similarly, we may associate to $d \omega$, which is still logarithmic, a current $T_{d \omega}$ of bidegree $(p+1,0)$. We then have, in the sense of currents,

$$
\partial T_{\omega}=T_{d \omega}
$$

which follows from the fact that if $\psi$ is any smooth $(0, p+1)$-form then $\int_{X} d(\omega \wedge \psi)=0$ by Stokes theorem (here $\omega \wedge \psi$ is again a current, and the integral is its value on $d 1 \equiv 0)$. In particular, we have

$$
\partial T_{\omega} \equiv 0 \Leftrightarrow d \omega=0 .
$$

On the other hand, $\bar{\partial} T_{\omega}$ is not zero: a simple computation, based on $\bar{\partial}\left(\frac{d z}{z}\right)=2 \pi i \delta_{0}$ (where $\delta_{0}$ is the Dirac distribution), shows that [No]

$$
\bar{\partial} T_{\omega}=2 \pi i T_{\operatorname{Res}(\omega)}
$$

where, with a negligible abuse of notation, we identify $T_{\operatorname{Res}(\omega)}$ (a current on $\hat{V}$ of bidegree $(p-1,0))$ with its direct image in $X$ (a current of bidegree $(p, 1))$.

By induction hypothesis, $\operatorname{Res}(\omega)$ is closed, i.e. $\partial T_{\operatorname{Res}(\omega)} \equiv 0$, and so $\bar{\partial} \partial T_{\omega} \equiv 0$. By regularity theory, the current $\partial T_{\omega}$ is in fact a holomorphic $(p+1)$-form, because it is of bidegree $(p+1,0)$ and $\bar{\partial}$-closed.

By Stokes Theorem,

$$
\int_{X} \partial T_{\omega} \wedge \overline{\partial T_{\omega}}=\int_{X} d\left(T_{\omega} \wedge \overline{\partial T_{\omega}}\right)=0
$$

( $T_{\omega} \wedge \overline{\partial T_{\omega}}$ is a current, being $\overline{\partial T_{\omega}}$ smooth, and its differential is $\partial T_{\omega} \wedge \overline{\partial T_{\omega}}$ because $\left.\bar{\partial} \partial T_{\omega} \equiv 0\right)$. This forces $\partial T_{\omega}$ to be identically zero, because $\partial T_{\omega} \wedge \overline{\partial T_{\omega}} \geq 0$.

Remark. We have used the projectivity of $X$ only to reduce the problem to $\operatorname{dim}_{\mathbf{C}}(X)=p+1$. In other words: any logarithmic $p$-form on any compact complex manifold of dimension $p+1$ is closed. 
In the context of manifolds with $\operatorname{Pic}(X)=\mathbf{Z}$, we shall use the following well-known fact:

Lemma 2.2. Let $X$ be a complex projective manifold with $\operatorname{Pic}(X)=\mathbf{Z}$ and $V \subset X$ a normal crossing hypersurface. Let $\omega$ be a global logarithmic $p$-form with poles along $V$ and $\operatorname{Res}(\omega) \not \equiv 0,1 \leq p \leq n-1$. Then $V$ is not smooth.

Proof: By contradiction, assume that $V$ is smooth, so that $\eta=\operatorname{Res}(\omega) \not \equiv$ 0 is a holomorphic $(p-1)$-form on $V$. The line bundle $\mathcal{O}_{X}(V)$ is ample, so that by Kodaira Vanishing Theorem $H^{p}\left(X, \mathcal{O}_{X}(-V)\right)=0$, because $p<n$. Hence the restriction map $H^{p-1}\left(X, \mathcal{O}_{X}\right) \rightarrow H^{p-1}\left(V, \mathcal{O}_{V}\right)$ is surjective.

The conjugate form $\bar{\eta}$ is a $(0, p-1)$-form on $V$ which is $\bar{\partial}$-closed, because $\eta$ is $\partial$-closed. Hence $\bar{\eta}$ defines a class in $H^{p-1}\left(V, \mathcal{O}_{V}\right)$ (à la Dolbeault), which arises from $H^{p-1}\left(X, \mathcal{O}_{X}\right)$, whence it follows that there exists a $\bar{\partial}$-closed $(0, p-1)$-form $\beta$ on $X$ whose restriction to $V$ is cohomologous to $\bar{\eta}$, that is equal to $\bar{\eta}+\bar{\partial} \gamma$ for some $(0, p-2)$-form $\gamma$ on $V$. After extending $\gamma$ to $X$ and replacing $\beta$ by $\beta-\bar{\partial} \gamma$, we may and will suppose that $\beta$ coincides with $\bar{\eta}$ on $V$.

Let now $\theta$ be a Kähler form on $X$. By considering $\eta$ as a current $T_{\eta}$ of bidegree $(p, 1)$ on $X$, we may evaluate it on the $(n-p, n-1)$-form $\theta^{n-p} \wedge \beta$ :

$$
T_{\eta}\left(\theta^{n-p} \wedge \beta\right)=\int_{V} \eta \wedge \theta^{n-p} \wedge \beta=\int_{V} \eta \wedge \bar{\eta} \wedge \theta^{n-p} .
$$

But $\theta^{n-p} \wedge \beta$ is $\bar{\partial}$-closed and $T_{\eta}=\frac{1}{2 \pi i} \bar{\partial} T_{\omega}$ is $\bar{\partial}$-exact, so that the integral is zero. Contradiction, because $\eta \wedge \bar{\eta} \wedge \theta^{n-p} \geq 0$ and $\eta \wedge \bar{\eta} \wedge \theta^{n-p} \not \equiv 0$.

It will be useful to reformulate Lemma 2.1 in a more abstract form, due to Bogomolov $[\mathbf{B o}]$, $[\mathbf{R e}]$. To this end, we recall the definition of Kodaira dimension of a holomorphic line bundle $L$ of a projective variety $X$, denoted $\kappa(X, L)[\mathbf{I i}]$. Consider the ring

$$
R(X, L):=\bigoplus_{m=0}^{\infty} H^{0}\left(X, L^{\otimes m}\right)
$$

and the homogeneous field of fractions $Q(X, L):=\left\{\frac{l_{i}}{l_{j}} \mid l_{i}, l_{j} \in H^{0}\left(X, L^{\otimes m}\right)\right.$, $m \geq 0\}$. Then we define $\kappa(X, L)$ as the transcendence degree of $Q(X, L)$, if $R(X, L) \neq \mathbf{C}$ or $\kappa(X, L):=-\infty$, if $R(X, L)=\mathbf{C}$. One has $\kappa(X, L) \leq$ $n=\operatorname{dim}_{\mathbf{C}}(X)$, and $\kappa(X, L)=n$ if $L$ is ample. 
Lemma 2.3. [Bo] Let $X$ be a complex projective $n$-manifold, $V \subset X$ a normal crossing hypersurface and $L \in \operatorname{Pic}(X)$. If there exists a nontrivial global section $\sigma$ of $\Omega_{X}^{p}(\log V) \otimes L$, then

$$
\kappa\left(X, L^{-1}\right) \leq p .
$$

Proof: Suppose by contradiction that $\kappa\left(X, L^{-1}\right) \geq p+1$, i.e. for some $m \geq 1$ there exist $p+2$ global sections $l_{0}, \ldots, l_{p+1} \in H^{0}\left(X, L^{\otimes-m}\right)$ such that the meromorphic functions on $X$ given by $f_{i}:=\frac{l_{i}}{l_{0}}$ are algebraically independent.

Let us suppose for a moment that $m=1$. We can multiply $\sigma$ by each $l_{i}$, obtaining global sections $\omega_{i}:=l_{i} \sigma \in H^{0}\left(\Omega_{X}^{p}(\log V)\right)$. Since $\omega_{i}=f_{i} \omega_{0}$, the closedness of each $\omega_{i}$ (Lemma 2.1) gives, for $i=1, \ldots, p+1$,

$$
d f_{i} \wedge \omega_{0} \equiv 0
$$

The nontriviality of $\omega_{0}$ implies $d f_{1} \wedge d f_{2} \wedge \ldots \wedge d f_{p+1} \equiv 0$, contradicting the algebraic independence.

The case $m>1$ is reducible to the case $m=1$ by passing to a suitable $m$-fold ramified covering. We refer to $[\mathbf{R e}]$ for details.

\section{Bounding the degree of solutions}

Let $\sigma \in H^{0}\left(X, \Omega_{X}^{p} \otimes N\right)$ be a Pfaff equation and $V \subset X$ a normal crossing hypersurface, which is a solution to $\sigma$.

We can look at $\sigma$ as a global holomorphic section of $\Omega_{X}^{p} \otimes \mathcal{O}_{X}(-V) \otimes$ $N \otimes \mathcal{O}_{X}(V)$, that is, a meromorphic section $\hat{\sigma}$ of $\Omega_{X}^{p} \otimes \mathcal{O}_{X}(-V) \otimes N$ with simple poles $(\hat{\sigma})_{\infty} \subset V$. Locally, i.e. after local trivialization of $\mathcal{O}_{X}(-V) \otimes N$, we can see $\hat{\sigma}$ as a meromorphic $p$-form $\omega$ with simple poles $(\omega)_{\infty} \subset V$ and we assert:

Lemma 3.1. The meromorphic $p$-form $\omega$ is logarithmic.

Proof: We have to check that that, if $\{f=0\}$ is a local reduced equation of $V$, then $d f \wedge \omega$ is holomorphic. From $i_{V} \sigma \equiv 0$ (Definition 1.2) it follows that $d f \wedge \sigma$ is identically zero along $V$, i.e.

$$
d f \wedge \sigma=f \cdot \theta
$$

for some regular local section $\theta$ of $\Omega_{X}^{p+1} \otimes N$. Hence $d f \wedge \frac{\sigma}{f}$ is a regular section of $\Omega_{X}^{p+1} \otimes N$ and $d f \wedge \omega$ is holomorphic, because (up to local trivialization) $\omega=\frac{\sigma}{f}$.

Remark that the converse of Lemma 3.1 is also true: if $\omega$ is a logarithmic $p$-form with polar set given by $\{f=0\}$, then $V=\{f=0\}$ is a solution to the Pfaff equation defined by $f \omega$. That's the reason for 
which the use of logarithmic forms is particularly well adapted to the study of solutions to Pfaff equations.

The meaning of Lemma 3.1 is that $\sigma$ is in fact a global holomorphic section of $\Omega_{X}^{p}(\log V) \otimes \mathcal{O}_{X}(-V) \otimes N$. Now, Bogomolov's Lemma (Lemma 2.3) gives:

$$
\kappa\left(X, \mathcal{O}_{X}(V) \otimes N^{-1}\right) \leq p<\operatorname{dim}_{\mathbf{C}}(X),
$$

which is the "bound on $d(V)$ " we were looking for.

Let us now specialize to the case $\operatorname{Pic}(X)=\mathbf{Z}$. Then

$$
\mathcal{O}_{X}(V) \otimes N^{-1}=l H,
$$

where $l=d(V)-d(N) \in \mathbf{Z}$ and $H$ is the positive generator of $\operatorname{Pic}(X)=$ Z. Since $l>0$ implies $\kappa(X, l H)=\operatorname{dim}_{\mathbf{C}}(X)$, we conclude from (3) that

$$
d(V)-d(N) \leq 0
$$

as desired. Moreover, $d(V)-d(N)=0$ means $\mathcal{O}_{X}(V) \otimes N^{-1}=\mathcal{O}_{X}$ and therefore the Pfaff equation is globally defined by a (closed!) logarithmic $p$-form $\omega$ with $(\omega)_{\infty}=V$ and hence $\operatorname{Res}(\omega) \not \equiv 0$. In this case, Lemma 2.2 says that $V$ is not smooth.

In order to apply this result to foliations of $\mathbf{C} P^{n}$, we remark:

Lemma 3.2. Let $\sigma \in H^{0}\left(\mathbf{C} P^{n}, \Omega_{\mathbf{C} P^{n}}^{p} \otimes N\right)$ be a Pfaff equation associated to a foliation $\mathcal{F}$ of $\mathbf{C} P^{n}$ with $\operatorname{codim}_{\mathbf{C}} \operatorname{Sing}(\mathcal{F}) \geq 2$ (and $\left.\operatorname{codim}_{\mathbf{C}}(\sigma)_{0} \geq 2\right)$. Then $d(N)=d(\mathcal{F})+p+1$.

Proof: Take a generic linear subspace $\Pi \simeq \mathbf{C} P^{p}$ and consider the restriction of $\sigma$ to $\Pi$, denoted $i_{\Pi} \sigma$, with hypersurface of zeros on $\Pi$ denoted by $\left(i_{\Pi} \sigma\right)_{0}$. Observe that $i_{\Pi} \sigma$ is a global regular section of $\left.K_{\mathbf{C} P^{p}} \otimes N\right|_{\Pi}$ vanishing on $\left(i_{\Pi} \sigma\right)_{0}$; hence

$$
\mathcal{O}_{\Pi}\left(\left(i_{\Pi} \sigma\right)_{0}\right)=\left.K_{\mathbf{C} P^{p}} \otimes N\right|_{\Pi},
$$

where $K_{\mathbf{C} P^{p}}$ is the canonical line bundle, whose degree is $-(p+1)$. Since $\left(i_{\Pi} \sigma\right)_{0}$ is the tangency set between $\mathcal{F}$ and $\Pi$ and $d(\mathcal{F})$ is defined as the degree of $\left(i_{\Pi} \sigma\right)_{0}$ in $\Pi$, we obtain $d(\mathcal{F})=-(p+1)+d(N)$.

Returning to the general inequality (3), we stress that it gives informations whatever $\operatorname{Pic}(X)$ is. Roughly speaking, it says that $\mathcal{O}_{X}(V)$ is "partially less positive" than $N$. First of all, let us observe that if $\kappa\left(X, \mathcal{O}_{X}(V) \otimes N^{-1}\right)=r \geq 0$ then (as the proof of Lemma 2.3 shows) on a suitable ramified covering of $X$ the Pfaff equation will be defined by a global (and closed) logarithmic $p$-form and will have $r$ algebraically independent first integrals. Next, when $X$ is a surface we have the following 
fact (probably, a similar statement holds in any dimension). Recall [Dm] that a divisor $D$ is nef if $D \cdot C \geq 0$ for every irreducible curve $C \subset X$.

Lemma 3.3. Let $M$ be a line bundle on a projective surface $X$ with $\kappa(X, M) \leq 1$. Then there exists a non-trivial nef divisor $D$, with real coefficients, such that $M \cdot D \leq 0$.

Proof: Let $N S_{\mathbf{R}}(X) \subset H^{2}(X, \mathbf{R})$ be the real Neron-Severi group of $X$, let $N_{\text {nef }} \subset N S_{\mathbf{R}}(X)$ be the nef cone (i.e., the closure of the ample cone), and let $N_{\text {psef }} \subset N S_{\mathbf{R}}(X)$ be the pseudoeffective cone (i.e., the closure of the effective cone). See for instance $[\mathbf{D m}]$ for these notions. Then, by Kleiman criterion (in its dual form), a line bundle $M$ belongs to the interior of $N_{\text {psef }}$ if and only if $M \cdot D>0$ for every $D \in N_{\text {nef }} \backslash\{0\}$. On the other hand, to say that $M$ belongs to the interior of $N_{\text {psef }}$ is the same as to say that $\kappa(X, M)=2$, by $[\mathbf{D m}$, Prop. 6.6]. Whence the result.

In our case, applied to $M=\mathcal{O}_{X}(V) \otimes N^{-1}$ this fact gives

$$
V \cdot D \leq N \cdot D
$$

On the other hand, one finds in $[\mathbf{B r}]$ the inequality

$$
V \cdot V \leq N \cdot V
$$

(and $V$ is not necessarily nef). The relation between these inequalities is not clear to us: one is a "global" statement about the line bundle $\mathcal{O}_{X}(V) \otimes N^{-1}$ over $X$, while the other is a "local" statement, i.e. about the restriction of $\mathcal{O}_{X}(V) \otimes N^{-1}$ to $V$.

We now discuss some possible extensions.

Given an analytic hypersurface $V \subset X$ whose singularities are worst than normal crossings, we can generalize the definition of logarithmic forms (Section 2) in two ways, which are not equivalent in general:

1) Given a meromorphic $p$-form $\omega$ with simple poles along $V=\{f=0\}$, we say that $\omega$ is weakly logarithmic if $f d \omega$ (or equivalently $d f \wedge \omega$ ) is holomorphic.

2) Given $\omega$ as in 1), we say that it is strongly logarithmic if, on a neighbourhood of any $x \in V, \omega$ belongs to the $\mathcal{O}_{X}$-module generated by holomorphic forms and the forms $\frac{d f_{1}}{f_{1}}, \ldots, \frac{d f_{k}}{f_{k}}$, where $V=$ $\left\{f_{1} \cdot f_{2} \ldots f_{k}=0\right\}$, with $f_{i}$ reduced equations of local branches of $V$ at $x$.

The previous Lemmata 2.1, 2.3 are still valid for strongly logarithmic forms, as was observed in $[\mathbf{N o}]$. There are at least two ways to see this: 
a) one can still define a good residue map for strongly logarithmic forms, and this is sufficient for the proof of Lemma 2.1

b) one can resolve the singularities of $V, \pi:(\tilde{X}, \tilde{V}) \rightarrow(X, V)$, and observe that the pull-back by $\pi$ of a strongly logarithmic form is (strongly) logarithmic.

On the other hand, Lemma 3.1 is still valid but the conclusion is only that $\omega$ is weakly logarithmic, which is clear from the proof.

It is exactly the discrepancy between weak and strong logarithmicity which is responsible for the failure of the inequality $d(V) \leq d(N)$ or $d(V) \leq d(\mathcal{F})+p+1$. For example, the foliation in $\mathbf{C} P^{2}$ given by $2 y d x-$ $5 x d y=0$ has a solution curve $V=\left\{x^{2}=y^{5}\right\}$ and $d(V)>d(\mathcal{F})+$ 2. The meromorphic 1-form $\omega=\frac{1}{x^{2}-y^{5}}(2 y d x-5 x d y)$ has simple poles along $V$ and defines $\mathcal{F}$. Observe that $\omega$ is weakly logarithmic, but not strongly logarithmic (and not closed). The reader may also verify that a sequence of blow-ups over 0 transforms $\omega$ to a meromorphic 1-form which is not logarithmic, in neither sense. Moreover, $\omega$ has a well-defined residue along $V \backslash\{0\}$, which is a holomorphic function on $V \backslash\{0\}$, but its extension to $V$ is only meromorphic (it has a pole of order two at 0 ). In other words, we cannot reasonably define a residue of $\omega$ along $V$, because such a residue should be a holomorphic function on $V$ (hence constant, if $V$ is compact).

These problems are related to the $G S V$ index studied in $[\mathbf{B r}]$. Let $X$ be a surface and $V \subset X$ be a curve, solution to a Pfaff equation $\sigma$, and let $\{f=0\}$ be a reduced equation of $V$ on a neighbourhood of $x \in V$. We work locally, hence we identify $\sigma$ with a holomorphic 1-form. Also, we suppose for a moment that $V$ is irreducible near $x$. We can find, on a neighbourhood of $x$, holomorphic functions $g$ and $h$, prime to $f$, and a holomorphic 1-form $\beta$ such that $g \sigma=h d f+f \beta$, i.e.

$$
\frac{\sigma}{f}=\frac{h}{g} \frac{d f}{f}+\frac{\beta}{g} .
$$

Again, $\frac{\sigma}{f}$ is weakly logarithmic but (possibly) not strongly logarithmic, for the (possible) presence of $g$. Outside $x \in V$ the 1 -form $\frac{\sigma}{f}$ has residue $\left.\frac{h}{g}\right|_{V \backslash\{x\}} \in \mathcal{O}_{V \backslash\{x\}}$. This residue has a meromorphic extension to $V$. Using the formula of the $G S V$ index given in $[\mathbf{B r}]$, we see that $G S V(\mathcal{F}, V, x)$ is equal to the vanishing order of $\frac{h}{g}$ at $x$ (which is negative if $\left.\frac{h}{g}\right|_{V}$ has a pole at $x$ ). Therefore, if $G S V(\mathcal{F}, V, x) \geq 0$ (which happens, for instance, if $x$ is a so-called non-dicritical singularity $[\mathbf{B r}])$, then the residue of $\frac{\sigma}{f}$ along $V \backslash\{x\}$ has a holomorphic extension to $V$. If $V$ has several irreducible local components $V_{1}, \ldots, V_{k}$ at $x$, one still finds that 
if $V$ is non-dicritical, then $\operatorname{Res}\left(\frac{\sigma}{f}\right) \in \mathcal{O}_{V \backslash\{x\}}$ has a holomorphic extension to $\bigoplus_{j=1}^{k} \mathcal{O}_{V_{j}}$ (each $\left.\operatorname{Res}\left(\frac{\sigma}{f}\right)\right|_{V_{j} \backslash\{x\}}$ extends holomorphically to $V_{j}$, but the vanishing order at $x$ of $\left.\operatorname{Res}\left(\frac{\sigma}{f}\right)\right|_{V_{j}}$ may depend on $j$ ). This is also related to the following fact, contained in the proof of Proposition 6 of $[\mathbf{B r}]:$ if $V$ is non-dicritical at $x$ and $\pi:(\tilde{X}, \tilde{V}) \rightarrow(X, V)$ is a resolution of $V$, then $\pi^{*}\left(\frac{\sigma}{f}\right)$ is a (strongly) logarithmic 1-form with poles along $\tilde{V}$. Consequently, inequality (3) still holds, i.e. $\kappa\left(X, \mathcal{O}_{X}(V) \otimes N^{-1}\right) \leq 1$, for $\operatorname{dim}_{\mathbf{C}}(X)=2$ and $V$ a non-dicritical separatrix ([Br, p. 533]).

Returning to the higher dimensional situation, let us observe that our bound $\kappa\left(X, \mathcal{O}_{X}(V) \otimes N^{-1}\right) \leq p$ obviously holds if $V \subset X$ has the property that every weakly logarithmic $p$-form with poles along $V$ is strongly logarithmic, by the previous considerations. This happens, for instance, if $V$ has (besides normal crossings) isolated singularities and $p \leq n-2$, by Saito's generalization of the De Rham division lemma $[\mathbf{S a}]$.

\section{References}

[Ba] E. BALlico, Meromorphic singular foliations on complex projective surfaces, Ann. Global Anal. Geom. 14(3) (1996), 257-261.

[Bo] F. A. Bogomolov, Unstable vector bundles and curves on surfaces, in: "Proceedings of the International Congress of Mathematicians" (Helsinki, 1978), Acad. Sci. Fennica, 1980, pp. 517-524.

[Br] M. BRunella, Some remarks on indices of holomorphic vector fields, Publ. Mat. 41(2) (1997), 527-544.

[Ca] M. M. CARnicer, The Poincaré problem in the nondicritical case, Ann. of Math. (2) 140(2) (1994), 289-294.

[CL] D. Cerveau and A. Lins Neto, Holomorphic foliations in $\mathbf{C} P(2)$ having an invariant algebraic curve, Ann. Inst. Fourier (Grenoble) 41(4) (1991), 883-903.

[De] P. Deligne, Théorie de Hodge. II, Inst. Hautes Études Sci. Publ. Math. 40 (1971), 5-57.

[Dm] J.-P. Demailly, $L^{2}$ vanishing theorems for positive line bundles and adjunction theory, in "Transcendental methods in algebraic geometry" (Cetraro, 1994), Lecture Notes in Math. 1646, Springer, Berlin, 1996, pp. 1-97.

[Ii] S. Ittaka, On D-dimensions of algebraic varieties, J. Math. Soc. Japan 23 (1971), 356-373.

[LN] A. Lins Neto, Some examples for poincaré problem and a question of Brunella, Preprint IMPA, 1999.

[No] J. Noguchi, A short analytic proof of closedness of logarithmic forms, Kodai Math. J. 18(2) (1995), 295-299. 
[Pa] P. Painlevé, "Oeuvres de Paul Painlevé. Tome I", Éditions du Centre National de la Recherche Scientifique, Paris, 1973.

[Po] H. Poincaré, Sur l'intégration algébrique des équations différentielles du 1er ordre et du 1er degré, Rend. Circ. Mat. Palermo 5 (1891), 161-191.

[Re] M. ReID, Bogomolov's theorem $c_{1}^{2} \leq 4 c_{2}$, in: "Proceedings of the International Symposium on Algebraic Geometry" (Kyoto Univ., Kyoto, 1977), Kinokuniya Book Store, 1978, pp. 623-642.

[Sa] K. SAITo, Theory of logarithmic differential forms and logarithmic vector fields, J. Fac. Sci. Univ. Tokyo Sect. IA Math. 27(2) (1980), 265-291.

[So] M. G. SoAREs, The Poincaré problem for hypersurfaces invariant by one-dimensional foliations, Invent. Math. 128(3) (1997), 495-500.

Laboratoire de Topologie

UMR 5584 CNRS

Université de Bourgogne

B.P. 47870 - 21078 Dijon

France

E-mail address: Marco.Brunella@u-bourgogne.fr

E-mail address: lgmendes@mat.ufrgs.br

Primera versió rebuda el 13 de desembre de 1999, darrera versió rebuda el 17 de maig de 2000. 